\title{
Tumour-suppressor genes prosper during asymmetric division
}

Asymmetric cell division is crucial during many developmental processes to generate two distinct cells from one mother cell. Occasionally this occurs through the activity of localized determinants in the progenitor cell, and nowhere is this process more clearly demonstrated than during neuroblast division in Drosophila melanogaster. In mitosis, the neural-fate determinants Prospero and Numb are localized to the basal area of the cell by their binding partners Miranda and Partner of Numb (PON), respectively. The localization of these determinants, and the apical localization of Inscutable (Insc) and Bazooka, which together control spindle orientation, ensure that the progenitor cell divides to give an apically derived neuroblast and a basally derived ganglion mother cell (GMC).

Recent work by Ohshiro et al. (Nature 408, 593-596; 2000) and Peng et al. (Nature 408, 596-600; 2000) has identified further genes that are involved in the localization of these basal determinants. Interestingly, these genes are two known Drosophila tumoursuppressor genes, lethal giant larvae (|gl), a WD-40-repeat protein, and discs large (dlg), a membrane-associated protein containing PDZ (PAR-6/Discs Large/ZO-1) domains.

Ohshiro and colleagues showed that embryos lacking both maternally and zygotically supplied copies of either $/ g\left(|g|^{G L C}\right)$ or $\mathrm{dlg}\left(\mathrm{dlg} \mathrm{gLC}^{\mathrm{GLC}}\right)$ do not localize Miranda to the basal membrane of neuroblasts, but instead exhibit uniform expression around the cortex (picture shows wild-type and Ig/GLC neuroblasts; Miranda is in green, $\alpha$-tubulin is in red). They also observed mislocalization of both Pon and Numb in /g/GLC neuroblasts, and mislocalization of Numb in dlgGLC neuroblasts. Peng and colleagues, in a similar study, found that /g/GLC or d/gGLC neuroblasts mislocalize Miranda and hence its binding partner Prospero, resulting in neuronal cell-fate defects. In wild-type neuroblasts, Dlg protein is expressed in an apical crescent that co-localizes with Bazooka and Insc, whereas Lg| protein is uniformly cortical. Without dlg, Lgl remains in the cytoplasm, which implicates Dlg in the control of Lgl localization. But how do these two tumour suppressors regulate asymmetric cell division?
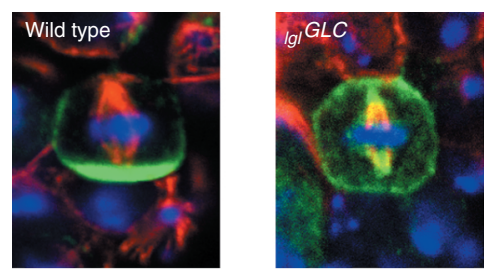

Previous work has shown that Igl homologues bind to non-muscle Myosin II proteins (Zipper in Drosophila). The authors of both recent studies have shown that Miranda is correctly localized in zipper mutants, yet zipper mutations can suppress the mislocalization of Miranda caused by Igl mutations. This indicates that high levels of myosin II may block the basal localization of Miranda when Lgl levels are low. However, the asymmetric localization of Pon does require myosin function. BDM, which is a general inhibitor of all myosins, suppresses the $/ g /$ mutant phenotype at low levels, which is consistent with the effects of zipper mutations. However, high concentrations of BDM mimic the lgl mutant phenotype. It is therefore likely that a different, unknown, myosin is positively required for the basal localization of Miranda.

These results clearly implicate Dlg and Lgl, and an as-yet-unknown myosin, in determining the basal localization of the determinants that are necessary for asymmetric cell division in an autonomous manner. Interestingly, in yeast and mammals, Lgl-related proteins have been shown to regulate secretory-vesicle fusion at specific membrane domains. Thus Lgl could act to move the basal determinants Miranda and Pon via the secretory system. However, Lgl may also promote actomyosin-dependent localization of determinants by affecting the activity of myosin II. Whatever the mechanism of protein targeting, it is highly significant that tumour-suppressor genes are involved in determining cell fate and division.

SARAH GREAVES 